Ann. Zootech., 1984, 33 (4), 557-562

\title{
Note
}

\section{Utilisation, en Guadeloupe, des techniques hormonales de maîtrise des cycles}

\author{
D. GAUTHIER *, G. COULAUD* et J. THIMONIER \\ I.N.R.A., Station de Recherches zootechniques \\ Centre de Recherches Antilles-Guyane \\ Domaine Duclos, F 97170 Petit-Bourg, Guadeloupe
}

\begin{abstract}
Résumé
L'utilisation des traitements hormonaux de maîtrise des cycles sexuels est testée sur des femelles de races laitière (FFPN) et allaitante (Créole) en Guadeloupe.

Sur les premières (102 femelles), le traitement (progestagène, valérate d'œstradiol, prostaglandine, PMSG et insémination artificielle 48 et 72 heures après la PMSG) permet d'obtenir une fertilité correcte entre les mois de décembre et mai $(55 \mathrm{p}$. 100) et faible le reste de l'année (18 p. 100).

Pour les femelles allaitantes (61), l'utilisation du traitement sans prostaglandine entraîne une fertilité moyenne de 49 p. 100 quelle que soit la saison. Si le nombre d'insémination est réduit à une (56 heures après la PMSG) la fertilité n'est pas diminuée (47 p. 100 sur 26 femelles).

Ces résultats montrent que les traitements hormonaux de maîtrise des cycles sont utilisables en Guadeloupe et devraient ainsi faciliter l'emploi de l'insémination artificielle.

Mots clés : maîtrise des cycles. bovins, zone tropicale.

\section{Introduction}

Les difficultés de détection des chalcurs en zone chaude chez les vaches laitières (Ortavant \& Loir, 1978) et l'existence de périodes d'anoestrus chez les femelles des races à viande, en particulier chez les bovins créoles (GauthiER \& Thimonier, 1982) limitent non seulement la reproduction mais également la diffusion du progrès génétique par l'insémination artificielle dans les pays tropicaux et notamment en Guadeloupe. C'est potrquoi, il nous a semblé important de contrôler dans ces. conditions d'élevage l'efficacité des techniques hormonales de maîtrise des cycles. mises au point en Europe (Chupin \& PELot, 1978).
\end{abstract} (France).

* Adresse actuelle : I.N.R.A., Laboratoire de la Production de Viande, Theix, 63122 Ceyrat. 


\section{Matériel et méthodes}

\section{A. Environnement}

Les expériences sont réalisées au domaine expérimental de la Station de recherches zootechniques de I'I.N.R.A. en Guadeloupe. La moyenne annuelle des précipitations est de $1280 \mathrm{~mm}$. Bien que d'importantes variations entre les années soient observées, une saison des pluies (août à novembre, $830 \mathrm{~mm}$ ) et une période sèche (décembre à juillet, $450 \mathrm{~mm}$ ) peuvent être définies. La température maximale moyenne varie entre $27^{\circ} \mathrm{C}$ (janvier) et $32^{\circ} \mathrm{C}$ (août), la température minimale entre $21^{\circ} \mathrm{C}$ et $25^{\circ} \mathrm{C}$. L'hygrométrie moyenne est toujours supérieure à $70 \mathrm{p} .100$.

\section{B. Animaux et traitemenis}

Les traitements sont appliqués à des bovins laitiers et allaitants.

\section{Conduite générale des troupeaux}

Les animaux de type laitier sont des femelles de race Française Frisonne Pie Noire ( $F F P N$ ) ; elles pâturent des savanes de Pangola (Digitaria decumbens) pendant la nuit et elles sont maintenues entravées et protégées du rayonnement solaire pendant la journée. Elles reçoivent alors une alimentation à l'auge, à base de fourrage vert ou ensilé (Pangola) et une complémentation en fonction de leur production laitière. Les génisses sont mises à la reproduction à un poids supérieur à $350 \mathrm{~kg}$.

Les animaux de type allaitant sont des femelles de race Créole (s'apparentant à Bos indicus) élevées toute l'année, sans abri, en savane à dominante de Petit Foin (Dicanthium caricosum). Le chargement est de l'ordre de $1400 \mathrm{~kg} / \mathrm{ha}$. La fumure est de 80 unités d'azote par hectare et par an. La durée d'allaitement des femelles est voisine de 7 mois. Les génisses sont mises à la reproduction à un poids supérieur à $250 \mathrm{~kg}$.

\section{Traitements}

a) $1^{\text {"n }}$ expérience

A deux saisons (janvier et juillet), 10 génisses FFPN et 10 génisses Créoles reçoivent ( $\mathrm{IO})$, par voie sous cutanée à la face externe de l'oreille, un implant contenant $6 \mathrm{mg}$ de Norgestomet (SC 21009 , Intervet). Au même moment, il est pratiqué, au niveau de l'encolure, une injection intramusculaire (IM) de $2 \mathrm{ml}$ d'une solution huileuse contenant $1,5 \mathrm{mg}$ de Norgestomet et $5 \mathrm{mg}$ de valerate d'oestradiol par $\mathrm{ml}$. Une injection de $25 \mathrm{mg}$ d'un analogue de la prostaglandine F2 $\alpha$ (Dinolytic, UPJOHN) est effectuée à $\mathrm{J} 7$ en IM dans l'encolure. A $\mathrm{I9}$, lors du retrait de l'implant, 400 UI de PMSG sont administrées (en IM) à chacune des femelles (Chupin \& Pelot, 1978) ; ce traitement constitue le traitement 1. Les chaleurs sont alors détectées toutes les 6 heures pendant 96 heures à l'aide d'un taureau vasectomisé.

Ce même traitement 1 est appliqué à un total de 102 vaches FFPN entre 40 et 60 jours post-partum, au cours de 3 années successives. Les inséminations artificielles 
sont réalisées systématiquement 48 et 72 heures après l'arrêt du traitement, sans détection des chaleurs.

\section{b) 2 expérience}

Le traitement 1, sans injection de l'analogue de la prostaglandine F2 $\alpha$ (traitement 2) est administré à 24 vaches $F F P N$ et 61 vaches Créoles qui sont aussi inséminées 48 et 72 heures après la fin du traitement.

\section{c) $3^{\circ}$ expérience}

Enfin, le traitement 2 est appliqué à 26 femelles Créoles (vaches et génisses) qui ne sont inséminées qu'une seule fois, 56 heures après l'injection de PMSG.

Pour l'ensemble de ces expériences, la fertilité est mesurée par le taux de mise bas. Pour les femelles Créoles, le taureau est introduit dans les lots 14 jours après l'injection de la PMSG de façon à saillir les vaches non gravides après insémination.

La durée de l'intervalle entre l'injection de la PMSG et le début des chaleurs a été étudiée par une analyse de variance de type split plot (GILL \& HAFS, 1971).

\section{Résultats}

\section{A. Intervalle fin traitement-oestrus (tabl. 1)}

Quelles que soient la saison et la race considérées, toutes les femelles présentent un comportement d'oestrus entre 24 et 96 heures suivant le retrait de l'implant. Mais il existe une interaction significative entre la saison et la race sur la durée de l'intervalle entre l'injection de PMSG et la venue en chaleurs; ainsi, cet intervalle est plus court en juillet qu'en janvier chez les génisses $F F P N(P<0,05 ; 43$ h vs 59 h) alors qu'il est constant chez les Créoles $(50 \mathrm{~h})$. Compte tenu de la durée moyenne des chaleurs induites ( $18 \mathrm{~h}$ chez les FFPN et $17 \mathrm{~h}$ chez les Créoles), le nombre maximum de femelles en chaleur est observé entre 48 et 72 heures après l'injection de la PMSG (72 p. 100 des femelles).

\section{B. Résultats d'utilisation sur les femelles FFPN (tabl. 2)}

La fertilité moyenne obtenue lors de l'oestrus induit avec le traitement 1 est de 41 p. 100. Mais il existe une variation saisonnière hautement significative. Ainsi, pendant la période chaude (août à novembre) la fertilité est de 18 p. 100, alors que de décembre à mai, elle est de 55 p. $100(\mathrm{P}<0,01)$. Lorsque le traitement est utilisé sans prostaglandine (traitement 2) la fertilité est égale à 54 p. 100 pendant la saison fraîche (décembre à mai), ce traitement n'a pas été testé en saison chaude.

\section{Résultats d'utilisation sur les femelles créoles (tabl. 3)}

La fertilité obtenue lors des inséminations réalisées 48 et 72 heures après PMSG est de 49 p. 100 et ne varie pas avec la saison. Si les inséminations sont effectuées uniquement 56 heures après PMSG la fertilité obtenue n'est pas différente de la précédente $(47$ p. 100). Toutefois, dans ce dernier cas, la fertilité des génisses (28 p. 100) est inférieure à celle des adultes $(60$ p. $100 ; \mathrm{P}<0,05)$. 


\section{Tableau 1}

Evolution cumulée du pourcentage de femelles présentant un astrus après un traitement d'induction et/ou synchronisation de l'ovulation.

Percentage of females exhibiting oestrus

after a hormonal treatment for ovulation induction and/or synchronization.

\begin{tabular}{|c|c|c|c|c|c|c|}
\hline \multicolumn{2}{|c|}{$\begin{array}{c}\text { Temps après la PMSG } \\
\text { Time after PMSG }\end{array}$} & $24-36 \mathrm{~h}$ & $36-48 \mathrm{~h}$ & $48-60 \mathrm{~h}$ & $60-72 \mathrm{~h}$ & $72-96 \mathrm{~h}$ \\
\hline Créoles & $\begin{array}{l}\text { Janvier }(10) \quad \ldots \\
\text { Juillet }(10) \ldots .\end{array}$ & $\begin{array}{r}0 \\
10\end{array}$ & $\begin{array}{l}20 \\
60\end{array}$ & $\begin{array}{l}50 \\
90\end{array}$ & $\begin{array}{r}70 \\
100\end{array}$ & $\begin{array}{l}100 \\
100\end{array}$ \\
\hline FFPN & $\begin{array}{l}\text { Janvier }(10) \\
\text { Juillet }(10)\end{array}$ & $\begin{array}{r}0 \\
30\end{array}$ & $\begin{array}{l}50 \\
40\end{array}$ & $\begin{array}{l}70 \\
60\end{array}$ & $\begin{array}{l}90 \\
80\end{array}$ & $\begin{array}{l}100 \\
100\end{array}$ \\
\hline
\end{tabular}

() Nombre de femelles - Number of females.

TABLEAU 2

Fertilité des femelles FFPN selon le type de traitement et la saison.

Fertility of FFPN females according to season and treatment.

\begin{tabular}{|c|c|c|c|c|}
\hline \multirow{4}{*}{$\begin{array}{l}\text { Implant }+ \text { PGF2 } \alpha+\text { PMSG } \\
2 \text { I.A. }(48-72 \text { h) } \\
\text { Implant + PMSG } \ldots \ldots \ldots \ldots \ldots \\
\text { 2 I.A. }(48-72 \text { h) }\end{array}$} & \multicolumn{3}{|c|}{$\begin{array}{l}\text { Fertilité en p. } 100 \\
\text { Fertility in p. } 100\end{array}$} & \\
\hline & \multicolumn{2}{|c|}{$\begin{array}{l}\text { Saison fraîche } \\
\text { Décembre - Mai } \\
\text { Fresh season }\end{array}$} & $\begin{array}{c}\text { Saison chaude } \\
\text { Août - Novembre } \\
\text { Hot season }\end{array}$ & \\
\hline & 55 & (64) & $18 \quad(38)$ & \multirow{2}{*}{$P<0001$} \\
\hline & \multicolumn{2}{|c|}{$54 \quad(24)$} & - & \\
\hline ( ) Nombre d'animaux - Number & animals. & & & \\
\hline
\end{tabular}

TABleau 3

Fertilité des femelles Créoles selon le rang de vêlage et le moment d'insémination.

Fertility of Creole females according to parity and insemination schedule.

\begin{tabular}{|c|c|c|c|}
\hline & & $\begin{array}{l}\text { Fertilité } \\
\text { Fertility }\end{array}$ & $\begin{array}{l}\text { n p. } 100 \\
\text { n p. } 100\end{array}$ \\
\hline $\begin{array}{l}\text { Implant + PMSG ... } \\
\text { (I.A. } 48 \text { et } 72 \text { heures) }\end{array}$ & $\begin{array}{c}\text { Vaches }+ \\
\text { génisses } \\
\text { Cow }+ \text { heifer }\end{array}$ & $49^{a}$ & $(61)$ \\
\hline $\begin{array}{l}\text { Implant + PMSG } \\
\text { (I.A. } 56 \text { heures) }\end{array}$ & $\begin{array}{l}\text { Vaches } \\
\text { Génisses }\end{array}$ & $\begin{array}{l}60^{u} \\
28^{13}\end{array}$ & $\begin{array}{l}(15) \\
(11)\end{array}$ \\
\hline
\end{tabular}

( ) Nombre de femelles - Number of females.

Les paramètres avec des indices différents sont significativement différents.

Parameters with different superscript are significantly different. 
En considérant l'oestrus induit et le premier retour en chaleur la fertilité de. l'ensemble des femelles est alors de 87 p. 100.

\title{
IV. Discussion et conclusion
}

La dynamique des venues en chaleur après le traitement hormonal confirme l'exactitude du choix des moments d'insémination et leurs similitudes avec ceux retenus en zone tempérée. Pour les vaches laitières, lorsque le traitement est appliqué à la saison favorable, la fertilité est du même ordre que celle obtenue par CHUPIN \& Pelot (1978) en Europe et supérieure à celle présentée par Jöchle et al. (1978) dans des conditions tropicales. En revanche, pendant la saison chaude, la faible efficacité de ce traitement nécessite l'utilisation conjointe de l'arrosage des animaux (Gauthrer, 1983) pour avoir une fertilité identique à celle obtenue en saison fraîche.

Pour les vaches allaitantes créoles, la fertilité de l'oestrus induit (environ 50 p. 100) doit permettre l'utilisation de l'insémination artificielle dans la pratique courante de l'élevage. De plus, l'insémination des vaches adultes, une seule fois, 56 heures après l'injection de PMSG est envisageable avec un taux de réussite satisfaisant.

Reçu en décembre 1983.

Accepté en août 1984.

\section{Remerciements}

Les auteurs remercient D. AgUer (Intervet, Angers) pour le don des produits de traitement et J. Pelot pour ses conseils dans la réalisation des protocoles.

\begin{abstract}
Summary
Hormonal treatments for controlling the sexual cycles of cows in Guadeloupe (French West Indies)
\end{abstract}

Hormonal treatments for control of sexual cycles were tested with dairy (FFPN) and bcef (Creole) cows in Guadeloupe (French West Indies).

In the dairy cows (102), the treatment (progestagen, oestradiol valerate, prostaglandin, PMSG and A.I. 48 and 72 hours after PMSG injection) led to fertility of 55 p. 100 between December and May and 18 p. 100 during the other months.

In the nursing cows $(61)$, the treatment without prostaglandin led to fertility of 49 p. 100 without any variation with the season or the insemination schedule and $47 \mathrm{p} .100$ if the insemination was performed only once 56 hours after PMSG injection (26 females).

Key words : control of cycles, cattle, tropical area. 


\section{Références bibliographiques}

Chupin D., Pelot J., 1978. Fertility of dairy cows treated with implants, protaglandin analog and PMSG. Theriogenology, 10, 307-311.

Gauthier D., Thimonier J., 1982. Variations saisonnières de la cyclicité chez la génisse Créole. Influences de la croissance, de l'âge et de l'émotivité. Reprod. Nutr. Develop., 22, 681-688.

Gauthier D., 1983. Technique permettant d'améliorer la fertilité des femelles Françaises Frisonnes Pie Noires (F.F.P.N.) en climat tropical. Influence sur l'évolution de la progestérone plasmatique. Reprod. Nutr. Develop., 23, 129-136.

Gill J.L., Hafs H.D., 1971. Analysis of repeated measurements of animals. J. Anim. Sci., 33, 331-336.

Jöchle W., Hidalgo M.A., Garcias R.C., 1978. Cycle synchronization and inductions in zebu cattle with chlormadinone acetate in differing dosage forms : effects of season. Zuchthuyg, 13, 21-27.

Ortavant R., LoIR M., 1978. The environment as a factor in reproduction in farm animals. 4th worla' Conf. anim. Prod., Buenos Aires, 1, 423-451. 